\title{
REGGE-SLOPE EXPANSION OF MESON BORN AMPLITUDES
}

P. H. Frampton* and Kameshwar C. Wali*

Physfics Department, Syracuse University, Syracuse, New York 13210

"Supported in part by the: U. S. Atomic Energy Commission

This report was prepared as an account of work sponsored by the United States Government. Neither the United States nor the United States Atomic Energy Commission, nor any of their employees, nor any of their contractors, subcontractors, or their employees, makes any warranty, express or implied, or assumes any

legal liability or responsibility for the accuracy, com

pleteness or usefulness of any information, apparatus, product or process disclosed, or represents that its use product or process disclosed, or represents
would not infringe privately owned rights. 


\section{DISCLAIMER}

This report was prepared as an account of work sponsored by an agency of the United States Government. Neither the United States Government nor any agency Thereof, nor any of their employees, makes any warranty, express or implied, or assumes any legal liability or responsibility for the accuracy, completeness, or usefulness of any information, apparatus, product, or process disclosed, or represents that its use would not infringe privately owned rights. Reference herein to any specific commercial product, process, or service by trade name, trademark, manufacturer, or otherwise does not necessarily constitute or imply its endorsement, recommendation, or favoring by the United States Government or any agency thereof. The views and opinions of authors expressed herein do not necessarily state or reflect those of the United States Government or any agency thereof. 


\section{DISCLAIMER}

Portions of this document may be illegible in electronic image products. Images are produced from the best available original document. 


\section{Abstract}

The expansion, in the Regge slope parameter $\alpha^{\prime}$; of simplified meson Born amplitudes comprising the Euler $B$ function and its multiparticle generalisation is considered in order to proceed towards a Lagrangian density $L$ which goes beyond the zero-slope-limit of scherk.

A discussion of the Laurent-Taylor expansion of the four-meson amplitude leads, at least formally, to a Lagrangian density in terms of a scalar field $\phi$; this density is, however, non-local since it contains an infinite number of derivatives. Certain approximation schemes, in terms of higher-mass poles of the full amplitude, are introduced.

Dimensionality arguments applied also to the multiparticle extension lead naturally to the expansion of $L$ in the Regge slope parameter, $\alpha^{\prime}$. The fuIl Lagrangian density is given, up to order $\alpha^{\prime 2}$ relative to the zero slope limit. At this order it is necessary to consider only the four-and five-point functions. 


\section{INTRODUCTION}

The zero-slope limit of the scalar boson Borm amplitudes in the dual resonance model was first investigated by Scherk ${ }^{l}$. He has shown that the four point amplitude represented by an Eulef Beta-function and the N-point amplitude represented by the generalisation of the beta-function reduce, in the zero-slope limit, to the conventional $\Phi^{3}$ Lagrangian theory. This demonstration and further work 2 along these lines is of considerable interest because they provide a linkage between the hadronic models on the one hand, and Lagrangian field theories on the other. Since the latter is considerably more fully explored and understood than the former, we may hope to learn a great deal from the connection.

The present work is aimed towards a further exploration of these ideas. We consider the expansion of a dual Bom amplitude in the slope parameter $\alpha$ ' and study the higher-order (non-linear) terms that would vanish in the limit considered by Scherk ${ }^{1}$. We investigate whether one can set up (even formally) a Lagrangian that can reconstruct the duality properties of the four-meson amplitude, and whether such a field theory is local. Thus in section II, we display the higher order terms in the four-meson amplitude represented by the Euler Beta-function and show how one can write a Lagrangian. We also discuss how various poles contribute to the higher order terms. In section III, multiparticle extensions of the beta function are considered with special emphasis on the five-point function. The final section IV is the summary and conclusions. Although we examine only the very simplest Born amplitudes - the beta-function and its generalisation to $\mathrm{N}$-particle amplitudes, it will be obvious that similar considerations can be applied to the more realistic Borm amplitudes now available. ${ }^{3}$

II. FOUR-MESON AMPLITUDE

Consider the elastic scattering amplitude $\mathrm{T}_{4}$ of four identical spinless particles with four momenta $p_{1}+p_{2} \rightarrow\left(-p_{3}\right)+\left(-p_{4}\right)$. Following Scherk ${ }^{1}$ we write 


$$
T_{4}=\left(g^{2} / 2\right)\left[B\left(-\alpha_{s},-\alpha_{t}\right)+B\left(-\alpha_{t},-\alpha_{u}\right)+B\left(-\alpha_{u},-\alpha_{s}\right)\right],
$$

where

$$
B(x, y)=\frac{\Gamma(x) \Gamma(y)}{\Gamma(x+y)}=\int_{0}^{1} d t \cdot t^{x-1}(1-t)^{y-1}
$$

and with $m$ as the mass of the external particle,

$$
\begin{aligned}
& x=-\alpha_{s}=-\alpha(0)-\alpha^{\prime} s=\alpha^{\prime}\left(m^{2}-s\right) ; \\
& y=-\alpha_{t}=-\alpha(0)-\alpha^{\prime} t=\alpha^{\prime}\left(m^{2}-t\right) .
\end{aligned}
$$

Now if we let $g, \alpha^{\prime} \rightarrow 0$ keeping $\lambda=g / \sqrt{\alpha^{\prime}}$ and $\mathrm{m}^{2}$ fixed (Scherk limit), we obtain

$$
B(x, y)=\frac{x+y}{x y} \frac{\Gamma(1+x) \Gamma(1+y)}{\Gamma(1+x+y)} \underset{x, y \rightarrow 0}{\rightarrow}\left(\frac{1}{x}+\frac{1}{y}\right),
$$

and

$$
\mathrm{T}_{4} \underset{\alpha \cdot \rightarrow 0}{=} \lambda^{2}\left(\frac{1}{\mathrm{~m}^{2}-\mathrm{s}}+\frac{1}{\mathrm{~m}^{2}-t}+\frac{1}{\mathrm{~m}^{2}-\mathrm{u}}\right)
$$

Physically, in the above limit, the masses of all the excited states go to infinlty leaving only the lowest mass ground state. It is not surprising then that $\mathrm{T}_{4}$ can be interpreted as the Born term arising from the Lagrangian

$$
\begin{aligned}
& L_{0}=L_{\text {free }}+\frac{1}{6} \lambda \phi^{3}, \\
& L_{\text {free }}=\frac{1}{2}\left((\partial \mu \phi)^{2}-\mathrm{m}^{2} \phi^{2}\right),
\end{aligned}
$$

which is the starting point of Scherk's work.

\section{A. Higher Order Terms}

To go beyond this result, we let $g, \alpha^{\prime}$ be non zero and consider the expansion in terms of $\lambda$ and $\alpha^{\prime}$. This can be achieved in several different ways. But the most straightforward way is to consider a Taylor Series expansion of the function

$$
E(x, y)=\frac{\Gamma(1+x) \Gamma(1+y)}{\Gamma(1+x+y)}=\sum_{r, s=0}^{\infty} D_{r s} x^{r} y^{s}
$$

in the region $|x|<1,|y|<1$. The expansion will converge in this domain whose projection on the plane Re $x$ - Re $y$ is shown in Fig. I. 
To evaluate the partial derivatives, we note that (putting $\phi(x, \dot{y})=\ln E(x, \dot{y})$ )

$$
\frac{\partial}{\partial x}(\ln E(x, y))=\phi_{x}(x, y)=[\psi(I+x)-\psi(I+x+y)]
$$

where $\psi(z)$ is the logarithmic derivative of the gamma function,

$$
\psi(z)=\frac{\Gamma^{\prime}(z)}{\Gamma(z)} \text {. }
$$

Likewise

$$
\frac{\partial}{\partial y}(\ln E(x, y))=\phi_{y}(x, y)=[\psi(I+y)-\psi(I+x+y)] .
$$

Then

$$
D_{r s}=D_{s r}=\left[\frac{1}{r !} \frac{1}{s !} \frac{\partial^{r}}{\partial x^{r}} \frac{\partial^{s}}{\partial y^{s}} E(x, y)\right]_{x=y=0} \text {. }
$$

In terms of the logarithmic derivatives $\phi_{x}(x, y), \phi_{y}(x, y)$ we find the following expression

$$
D_{r s}=\frac{1}{r ! s !} E(x, y)\left[\left(\phi_{x}+\frac{\partial}{\partial x}\right)\left(\phi_{y}+\frac{\partial}{\partial y}\right) 1\right]_{x=y=0} .
$$

This is convenient for direct evaluation of the Taylor coefficients, care being necessary to remark the non-commutativity of the operators. We can obtain an explicit formal expression for $D_{\text {rs }}$ in the following way:

$$
\frac{\partial^{s}}{\partial y^{s}} E(x, y)=E(x ; y) \sum_{\substack{m=0 \\ \sum a_{K}=m}}^{s k a_{K}=s}
$$

with the partition coefficients (e.g. Ref. 4)

$$
\left(s ; a_{1}, a_{2}, \ldots a_{s}\right)=\frac{s !}{(11)^{a_{1}} a_{1} !(2 !)^{a_{2}} a_{2} ! \ldots(s !)^{a_{s}} a_{s} !}
$$

We note from (2.9) and (2.11) that

$$
\begin{aligned}
& {\left[\frac{\partial^{r}}{\partial x^{r}} \phi_{x}(x, y)\right]_{x=y=0}=\left[\frac{\partial^{r}}{\partial y} \phi_{y}(x, y)\right]_{x=y=0}=0,} \\
& {\left[\frac{\partial^{r}}{\partial y^{r}} \phi_{(k+I) x}(x, y)\right]_{x=y=0}=\left[\frac{\partial^{r}}{\partial y^{r}} \phi_{(k+I) y}(x, y)\right]_{x=y=0}=-\psi^{(r+k)} \cdot(I) .}
\end{aligned}
$$

Then 


$$
\left.\frac{\partial^{r}}{\partial x^{r}} E(x, y)\right|_{x=y=0}=0
$$

and

$$
\begin{aligned}
& \frac{\partial^{r}}{\partial x^{r}}\left[\left(\phi_{y}\right)^{a_{1}}\left(\phi_{y y}\right)^{a_{2}} \ldots\left(\phi_{s y}\right)^{a_{s}}\right] \\
& \left.=\sum_{r_{1} r_{2} \cdots r_{s}} \frac{r !}{r_{1} ! r_{2} ! \ldots r_{s} l}\left(\frac{\partial^{r_{1}}}{\partial x^{r_{1}}}\left(\phi_{y}\right)^{a_{1}}\right)\left(\frac{\partial^{r_{2}}}{\partial r^{r_{2}}}\right)\left(\phi_{y y}\right)^{a_{2}}\right) \ldots\left(\frac{\partial^{r_{s}}}{\partial x^{r_{s}}}\left(\phi_{s y}\right)^{a_{s}}\right) . \\
& \sum_{k=1}^{s} r_{k}=r
\end{aligned}
$$

Hence the nonvanishing $D_{r s}$ are given by

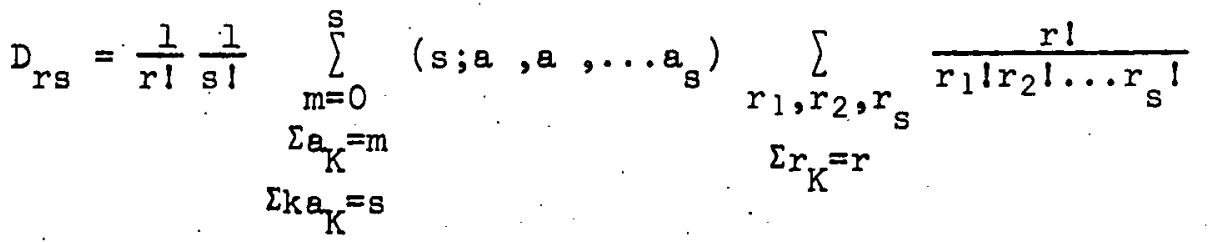

$$
\begin{aligned}
& {\left[\left(\frac{\partial^{r_{1}}}{\partial x^{r_{1}}}\left(\phi_{y}\right)^{a_{1}}\left(\frac{\partial^{r_{2}}}{\partial x^{r_{2}}}\left(\phi_{y y}\right)^{a_{2}}\right) \ldots\left(\frac{\partial^{r_{s}}}{\partial x^{r_{s}}}\left(\phi_{s y}\right)^{a^{s}}\right)\right]_{x=y=0}\right.}
\end{aligned}
$$

where

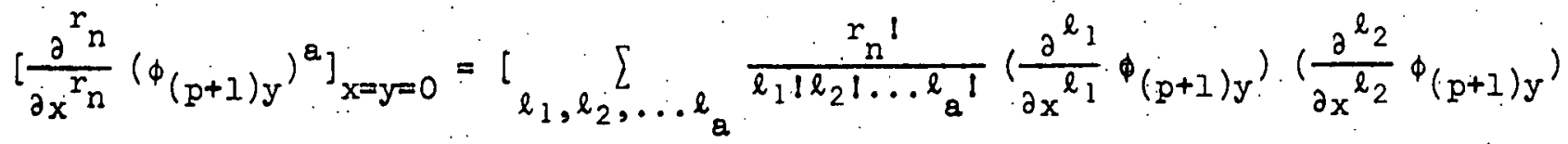

$$
\begin{aligned}
& \Sigma l_{i}=r_{n} \\
& \left.\ldots\left(\frac{\partial^{l_{a}}}{\partial x^{l_{a}}} \phi(p+1) y\right)\right]_{x=y=0}
\end{aligned}
$$

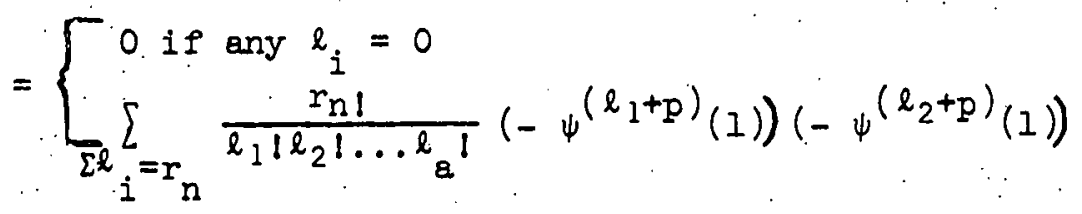

$$
\begin{aligned}
& \text {... }\left(-\psi^{\left(l_{a}+p\right)}(1)\right), \\
& \text { if every } \ell_{i} \geq 1 \text {. }
\end{aligned}
$$

It is seen that all the $D_{\text {rs }}$ (related to the higher order coupling constants of the Lagrangian, discussed below) can be fully expressed in terms of derivatives, 
up to order $(r+s-1)$ of the psi function, taken with unit argument. The first few terms are as follows

$$
\begin{aligned}
E(x, y)= & I-x y\left\{\psi^{(1)}+\frac{1}{2 !} \psi^{(2)}(x+y)+\frac{1}{3 !} \psi^{(3)}\left(x^{2}+y^{2}\right)\right. \\
& \left.-\frac{1}{2}\left(\psi^{(1)}\right)^{2}-\frac{1}{2 !} \psi^{(3)}\right) x y+\frac{1}{4 !} \psi^{(4)}\left(x^{3}+y^{3}\right) \\
& -\frac{1}{2}\left(\psi^{(1)} \psi^{(2)}-\frac{1}{3 !} \psi^{(4)}\right) x y(x+y) \\
& +\frac{1}{5 !} \psi^{(5)}\left(x^{4}+y^{4}\right)-\frac{1}{2}\left(\frac{1}{3} \psi^{(1)} \psi^{(3)}+\frac{1}{4}\left(\psi^{(2)}\right)^{2}-\frac{1}{4 !} \psi^{(5)}\right) x y\left(x^{2}+y^{2}\right) \\
& -\frac{1}{3 !}\left(-\left(\psi^{(1)}\right)^{3}+\frac{3}{2}\left(\psi^{(2)}\right)^{2}+\frac{3}{2} \psi^{(1)} \psi^{(3)}-\frac{1}{3 !} \psi^{(5)}\right) x^{2} y^{2\}}+0\left(\alpha^{7}\right),(2
\end{aligned}
$$

where $\psi^{(m)}=\psi^{(m)}(1)$. Then

$$
\begin{aligned}
B(x, y)= & \left(\frac{1}{x}+\frac{1}{y}\right)-\psi^{(1)}(x+y)-\frac{1}{2 l} \psi^{(2)}(x+y)^{2} \\
& -\frac{1}{3 !} \psi^{(3)}(x+y)^{3}+\frac{1}{2}\left(\left(\psi^{(1)}\right)^{2}+\frac{1}{3 !} \psi^{(3)}\right) x y(x+y)+0\left(\alpha^{4}\right)
\end{aligned}
$$

where we have noted that $x, y^{\prime} \sim \alpha^{\prime}$.

When we sum over the permutations implied by (2.1), the contribution of the first nonsingular term to $\mathrm{T}_{4}$ is given by

$$
8^{2} \alpha \cdot\left[3 m^{2}-(s+t+u)\right] \psi(1)=8^{2} \alpha \cdot\left[-3 m^{2}+\sum_{\substack{i, j=1 \\ 1 \neq 1}}^{4} p_{i} \cdot p_{j}+\frac{3}{2} \sum_{i,=1}^{4} p_{i}{ }^{2}\right] \psi(1) .
$$

To obtain this contribution from a Lagrangian, we have to add to (2.6) an additional piece ${ }^{5} L_{2}$ where

$$
L_{2}=-\psi(1) \lambda^{2} \alpha^{\prime 2}\left[\frac{1}{4}(\partial \mu \phi)^{2} \phi^{2}+\frac{1}{4}\left(\partial \mu^{2} \phi\right) \phi^{3}+\frac{1}{8} \dot{m}^{2} \phi^{4}\right]:
$$

It should be noted that we have deliberately not set $p_{i}{ }^{2}=\mathrm{m}^{2}$. If the mass shell condition is used, $(2.23)$ is simply $\left(\lambda^{2} \alpha^{\prime 2} \mathrm{~m}^{2} \psi^{(1)}\right)$ which can be derived, from any of the following three forms of the Lagrangian $L_{2}$

$$
\psi^{(1)} \lambda^{2} \alpha^{\prime 2}\left[+\frac{1}{8}(\partial \mu \phi)^{2} \phi^{2}\right], \psi^{(1)} \lambda^{2} \alpha^{\prime} 2\left[-\frac{1}{24}\left(\partial \mu^{2} \phi\right) \phi^{3}\right] \text { or } \frac{1}{24} \psi^{(1)} \lambda^{2} \alpha^{\prime 2} \mathrm{~m}^{2} \phi^{4} \text {. }
$$

These altemate forms of the Lagrangian which give identical results as far as the four point function is concemed will not lead to the same result when we 
consider the five-point function. We shall see this explicitly in the next section.

It is clear that we can, proceeding in this manner, write a Lagrangian density which reproduces any or all of the terms in the series expansion of $B(x, y)$. In principle, therefore, we can reconstruct the dual amplitude in two steps, as follows:

(i) Go to a kinematical region where $\left|\alpha_{s}\right|,\left|\alpha_{t}\right|,\left|\alpha_{u}\right|<1$. Use the full Lagrangian (containing arbitrarily many derivatives acting on $\phi^{4}$ ) to deduce the S-matrix in a Born-approximation.

(11) This will reproduce, accurately, $B\left(-\alpha_{s},-\alpha_{t}\right)$ which may then be analytically continued to all $\alpha_{s}$, $\alpha_{t}$ from its defining functional form.

B. Higher Mass Resonance Poles and Higher Order Terms

As remarked at the beginning of this section, in the limit, $g, \alpha^{\prime} \rightarrow 0$, all the higher mass states in $B(x, y)$ go to infinity. It is instructive to consider their contributions when $g, \alpha^{\prime} \neq 0$.

For this purpose consider the expension of $E(x, y)$ in terms of t-channel poles:

$$
E(x, y)=\sum_{N=1}^{\infty}(-1)^{N-1} \cdot \frac{\Gamma(1+x)}{(N-1) ! \Gamma(x-N+1)} \frac{1}{y+N}
$$

Now

$$
\frac{1}{y+N}=\sum_{p=0}^{\infty}(-1)^{p} \frac{1}{N}\left(\frac{y}{N}\right)^{p}
$$

Hence

$$
E(x, y)=1-\sum_{N=1}^{\infty} \sum_{p=1}^{\infty}(-1)^{p} \frac{\Gamma(N-x)}{\Gamma(-x)} \frac{1}{N ! N} y^{p} .
$$

Therefore,

$$
\left.\frac{\partial E(x, y)}{\partial x}\right|_{x=0}=\sum_{p=1}^{\infty}(-1)^{p} y^{p} \sum_{N=1}^{\infty} \frac{1}{N^{p+1}}=\sum_{p=1}^{\infty}(-1)^{p} y^{p} \zeta(p+1),
$$

where $\zeta(z)$ is the Riemann zeta function. 
From $(2.8)$

$$
\left.\frac{\partial E}{\partial x}\right|_{x=0}=\psi(1)-\psi(1+y)=-\sum_{p=1}^{\infty} \frac{\psi^{(p)}}{p l} y^{p}=\sum_{p=1}^{\infty} D_{1 p} y^{p}
$$

From the 1mportant identity

$$
(-1)^{p} \zeta(p+1)=-\frac{\psi(p)}{p !}
$$

we have

$$
D_{1 p}=-\frac{\psi^{(p)}}{p !}=(-1)^{p} \zeta(p+1)=(-1)^{p} \cdot \sum_{N=1}^{\infty} \frac{1}{N^{p+1}},
$$

which shows how individual poles contribute to $D_{1 p}$ which is the coefficient of $(x+y)^{P}$ in the expansion (2.22) of $B(x, y)$. Thus the contribution of the $N^{\text {th }}$ pole (1.e. $y=-N$ or $\alpha(t)=N$ ) to $\psi(p) / p$ i falls off as $\frac{1}{N^{p+1}}$. This suggests an approximation scheme in which

$$
\frac{\psi^{(p)}}{p !} \approx(-i)^{p+1}
$$

1.e. we retain only the $N=1$ pole. In this case

$$
E(x, y)=1+x \sum_{p=1}^{\infty}(-1)^{p} y^{p}=1-x+\frac{x}{1+y}
$$

and hence

$$
B(x, y) \approx\left(\frac{1}{x}+\frac{1}{y}\right)-\frac{(x+y)}{(1+y)}
$$

From the point of view of writing an equivalent Lagrangian, equs. (2.34) and (2.35) are unsuitable as they are manifestly not cyclic symmetric. If we use the approximation (2.33) in (2.19), we are led to the more interesting form

$$
E(x, y)=1-\frac{x y}{(1+x)(1+y)}
$$

and

$$
B(x, y)=\left(\frac{1}{x}+\frac{1}{y}\right)-\frac{x+y}{(1+x)(1+y)} .
$$


It is clear that even in this approximation the Lagrangian corresponding to (2.37) and 1ts permutations written in terms of $\phi$ contains an infinite number of terms ${ }^{6}$ involving derivatives of $\phi$. Thus it is only in the strict $g, \alpha^{\prime}+0$ limit, one has a simple renormalizable local Lagrangian, although another possible approximation scheme, this time in $\alpha^{\prime}$, will be discussed in the next section.

To conclude this section, we note that, instead of expanding around the poles $\alpha_{g}=\alpha_{t}=0$, we can alternatively expand around any arbitrary pole. Agaln we can separate the non-pole terms and write expansions similar to (2.20) and (2.21). This does not shed any new light on the structure of the Lagrangian. III. MULTIPARTICLE EXTENSION

In the zero-slope limit, $B$ and $\alpha^{\prime} \rightarrow 0$ the simple $\lambda \phi^{3}$ Lagrangian of Eq. (2.6), cound from the four-meson amplitude faithfully reproduces the corresponding limt of the multiparticle amplitudes provided that one normalized the multiparticle T-matrix by

$$
\begin{gathered}
T_{N}=\sum_{\begin{array}{c}
\text { inequivalent } \\
\text { permutations }
\end{array}} F_{n}\left(p_{1} p_{2} \cdots p_{N}\right) \\
F_{N}\left(p_{1} p_{2} \cdots p_{N}\right)=\frac{B^{N-2}}{2^{N-3}}\left(\alpha^{\prime}\right)^{\frac{1}{2}(N-4)} B_{N}\left(p_{1} p_{2} \cdots p_{N}\right) .
\end{gathered}
$$

The powers of $\alpha^{\prime}$ in ine definition of, $F_{N}$ is essential from dimensionality conslderations; when we use covariant normalizations for the external wave functions. The numerical factor $2^{3-N}$ is such that the Feynman-Born diagrams all appear with the correct coefficient at each $\mathrm{N}$ value.

The question arises: Once we know the higher terms in the Regge slope expansion at the four-mesor level, cerivable from $\phi^{4}$ interaction terms as discussed In the previous section, is this again sufficient to reproduce accurately the R:gge slope expansions of the multiparticle dual amplitudes. 


\section{A. Types of Terms which may Occur.}

On the grounds of dimensionality alone, it is straightforward to discuss the possible interaction terms which might be necessary to reproduce the multiparticle amplitudes. Taking as the fundamental dimension that of mass (M) we note that the scalar field has dimension $[\phi]=M$, the zero-slope coupling has dimension $[\lambda]=\left[\delta \alpha^{\alpha^{-\frac{1}{2}}}\right]=M$ and for the Lagrangian density itself $[L]=M^{4}$.

The possible terms in the Lagrangian density are listed in Table 1 . It is convenient to refer to the simplest (scherk) term $\lambda \phi^{3}$ as the zero-slope limit, to the terms $\lambda^{\mathbb{N}-2}\left(\alpha^{\prime}\right)^{N-2} \cdot \phi^{\mathbb{N}}(\mathbb{N} \geq 4)$ as contact terms ${ }^{7}$ and to all other terms (necessarily of higher order in $\alpha^{\prime}$ ) as derivative terms. These derivative terms must contain either derivatives or powers of $\mathrm{m}^{2}$, or both, acting on $\phi^{\mathrm{N}}$.

The pattem of terms that are allowed by dimensionality will suggest an approximation where we truncate the Lagrangian $L$, at a definite order in $\alpha^{\prime}$ relative to the zero-slope limit.

B. The Contact Term for $N=5$.

We now consider the $B_{5}\left(p_{1} p_{2} p_{3} p_{4} p_{5}\right)$ Born amplitude for a fixed permutation of the external legs, and attempt to write for it a Laurent expansion in the five complex variables $x_{i}=-\alpha_{i, i+1}=-\alpha(0)-\alpha^{\prime}\left(p_{i}+p_{i+1}\right)^{2}$.

It is particularly convenient to separate out explicitly the known single and double ground-stote poles of $B_{5}$ in order to extract a function $E_{5}\left(\begin{array}{lllll}x_{1} & x_{2} & x_{3} & x_{4} & x_{5}\end{array}\right)$ which is analytic in $\left|x_{i}\right|<1$. The analytic function $E_{5}$ plays a role analogous to that of the function $E(x, y)$ at the four-meson level. It seems to be impossible to separate such a function multiplicatively from $B_{5}$, but it can be separated additively. To be specific we write 


$$
\begin{aligned}
B_{5}\left(p_{1} p_{2} p_{3} p_{4} p_{5}\right)= & \frac{1}{x_{1}} B_{4}\left(x_{3} x_{4}\right)+\frac{1}{x_{2}} B_{4}\left(x_{4} x_{5}\right)+\frac{1}{x_{3}} B_{4}\left(x_{5} x_{1}\right) \\
& +\frac{1}{x_{4}} B_{4}\left(x_{1} x_{2}\right)+\frac{1}{x_{5}} B_{4}\left(x_{2} x_{3}\right) \\
& -\frac{1}{x_{1} x_{3}}-\frac{1}{x_{2} x_{4}}-\frac{1}{x_{3} x_{5}}-\frac{1}{x_{4} x_{6}}-\frac{1}{x_{5} x_{1}} \\
\therefore & +E_{5}\left(x_{1} x_{2} x_{3} x_{4} x_{5}\right)
\end{aligned}
$$

which defines $E_{5}$. This definition, together with the internal bootstrap property of $B_{5}$, engures that $E_{5}\left(x_{1} x_{2} x_{3} x_{4} x_{5}\right)$ is analytic inside $\left|x_{i}\right|<1, i=1,2,3,4,5$.

The question of whether a $\lambda^{3} \alpha^{12} \phi^{5}$ contact term must be present in $L$ is now seen to be equivalent to asking whether the analytic function $\mathrm{E}_{5}$ has a non-vanishing zeroth-order term in its five-fold Taylor expansion around $x_{i}=0$.

Let us therefore examine carefully the double-integral representation 8 $B_{5}\left(p_{1} p_{2} p_{3 p_{4}} p_{5}\right)=\int_{0}^{1} d t_{1} \int_{0}^{1} d t_{2} t_{1}^{x_{1}-1}\left(\frac{1-t_{1}}{1-t_{1} t_{2}}\right)^{x_{2}-1}\left(\frac{1-t_{2}}{1-t_{1} t_{2}}\right)^{x_{3}-1} t_{2} x_{4}-1$

$$
\left(1-t_{1} t_{2}\right)^{x_{5}-2}
$$

and divide up the domain of integration as indicated in Figure 2(a). The boundaries are defined by

$$
\begin{array}{ll}
\text { 1. } & 0 \leq t_{1}, t_{2} \leq \varepsilon \\
\text { 2. } & 0 \leq t_{2} \leq \varepsilon ; \quad \varepsilon \leq t_{1} \leq(1-\varepsilon) \\
\text { 3. } & 0 \leq t_{1} \leq \varepsilon ; \quad \varepsilon \leq t_{2} \leq(1-\varepsilon) \\
\text { 4. } & 0 \leq\left(1-t_{1}\right), t_{2} \leq \varepsilon \\
\text { 5. } & 0 \leq t_{1},\left(1-t_{2}\right) \leq \varepsilon \\
\text { 6. } & \varepsilon \leq t_{2} \leq(1-\varepsilon) ;(1-\varepsilon)\left(1-\varepsilon t_{2}\right)^{-1} \leq t_{1} \leq 1 \\
\text { 7. } & \varepsilon \leq t_{1} \leq(1-\varepsilon) ;(1-\varepsilon)\left(1-\varepsilon_{1}\right)^{-1} \leq t_{2}-1 \\
\text { 8.9.10. } \quad(1-\varepsilon) \leq t_{1} t_{2} \leq 1
\end{array}
$$

where $\varepsilon \geq 0$ is a small positive number. The final region contains three distinct singularities which may be further separated by defining 


$$
\begin{aligned}
& t_{1}=1-u_{1} u_{2} \\
& t_{2}=\frac{1-u_{1}}{1-u_{1} u_{2}}
\end{aligned}
$$

and defining the sub-regions (Fig. 2(b))

$$
\begin{aligned}
& \text { 8. } 0 \leq u_{1}, u_{2} \leq \varepsilon \\
& \text { 9. } 0 \leq u_{1} \leq \varepsilon ; \quad \varepsilon \leq u_{2} \leq(1-\varepsilon) \\
& \text { 10. } 0 \leq u_{1},\left(1-u_{2}\right) \leq \varepsilon .
\end{aligned}
$$

It is now straightforward to check that these ten contributions are given by

$$
\begin{aligned}
& \left(\frac{1}{x_{1}}+\varepsilon_{1}\right)\left(\frac{1}{x_{4}}+\varepsilon_{4}\right)+\left(\frac{1}{x_{2}}+\varepsilon_{2}\right)\left(\frac{1}{x_{5}}+\varepsilon_{5}\right) \\
& +\left(\frac{1}{x_{3}}+\varepsilon_{3}\right)\left(\frac{1}{x_{5}}+\varepsilon_{5}\right)+\left(\frac{1}{x_{4}}+\varepsilon_{4}\right)\left(\frac{1}{x_{1}}+\varepsilon_{1}\right) \\
& +\left(\frac{1}{x_{5}}+\varepsilon_{5}\right)\left(\frac{1}{x_{2}}+\varepsilon_{2}\right)+\left(\frac{1}{x_{1}}+\varepsilon_{1}\right) \tilde{B}_{4}\left(x_{3} x_{4}\right) \\
& +\left(\frac{1}{x_{2}}+\varepsilon_{2}\right) \tilde{B}_{4}\left(x_{4} x_{5}\right)+\left(\frac{1}{x_{3}}+\varepsilon_{3}\right) \tilde{B}_{4}\left(x_{5} x_{1}\right) \\
& +\left(\frac{1}{x_{4}}+\varepsilon_{4}\right) \tilde{B}_{4}\left(x_{1} x_{2}\right)+\left(\frac{1}{x_{5}}+\varepsilon_{5}\right) \tilde{B}_{4}\left(x_{2} x_{3}\right)
\end{aligned}
$$

where we have made the definitions:

$$
\varepsilon_{i}=\int_{0}^{\varepsilon} d t t^{x_{i}-1}-\frac{1}{x_{i}}=\frac{\varepsilon^{x_{i}}-1}{x_{i}}
$$

for $1=1,2,3,4,5$ and

$$
\begin{aligned}
\tilde{B}_{4}(x, y) & =\int_{\varepsilon}^{1-\varepsilon} d t t^{x-1}(1-t)^{y-1} \\
& =B_{4}(x, y)-\frac{1}{x}-\frac{1}{y}-\varepsilon_{x}-\varepsilon_{y} .
\end{aligned}
$$

Combining terms we may therefore re-write expression (3.18) as 


$$
\begin{aligned}
& \frac{1}{x_{1}} B_{4}\left(x_{3} x_{4}\right)+\frac{1}{x_{2}} B_{4}\left(x_{4} x_{5}\right)+\frac{1}{x_{3}} B_{4}\left(x_{5} x_{1}\right)+\frac{1}{x_{4}} B_{4}\left(x_{1} x_{2}\right)+\frac{1}{x_{5}} B_{4}\left(x_{2} x_{3}\right) \\
& -\frac{1}{x_{1} x_{3}}-\frac{1}{x_{2} x_{4}}-\frac{1}{x_{3} x_{5}}-\frac{1}{x_{4} x_{1}}-\frac{1}{x_{5} x_{2}} \\
& +\varepsilon_{1}\left(B_{4}\left(x_{3} x_{4}\right)-\frac{1}{x_{3}}-\frac{1}{x_{4}}\right)+\varepsilon_{2}\left(B_{4}\left(x_{4} x_{5}\right)-\frac{1}{x_{4}}-\frac{1}{x_{5}}\right) \\
& \therefore \\
& +\varepsilon_{3}\left(B_{4}\left(x_{5} x_{1}\right)-\frac{1}{x_{5}}-\frac{1}{x_{1}}\right)+\varepsilon_{4}\left(B_{4}\left(x_{1} x_{2}\right)-\frac{1}{x_{1}}-\frac{1}{x_{2}}\right) \\
& \quad \\
& +\varepsilon_{5}\left(B_{4}\left(x_{2} x_{3}\right)-\frac{1}{x_{2}}-\frac{1}{x_{3}}\right) \\
& \quad \\
& -\varepsilon_{1} \varepsilon_{3}-\varepsilon_{2} \varepsilon_{4}-\varepsilon_{3} \varepsilon_{5}-\varepsilon_{4} \varepsilon_{1}-\varepsilon_{5} \varepsilon_{2}
\end{aligned}
$$

In the limit $\varepsilon+0+1$ is obvious that

$$
\varepsilon_{1}+\ln \varepsilon \quad(\rightarrow \infty) \text {. }
$$

Hence these regions of integration give a contribution to $B_{5}\left(x_{1} x_{2} x_{3} x_{4} x_{5}\right)$ that is

$$
-5(\ln \varepsilon)^{2}
$$

There is no $\varepsilon$-independent $x_{i}$-independent term from these regions. The divergence (3.24) must be cancelled by a corresponding term in the non-singular region: We may easily calculate the latter, in the limit $x_{i} \rightarrow 0$, from its expression

$$
\begin{aligned}
& \left(\int_{\varepsilon}^{1-\varepsilon} d t_{1} \int_{\varepsilon}^{1-\varepsilon} d t_{2}+\int_{1-\varepsilon}^{1} d t_{1} \int_{1-\varepsilon}^{\frac{1-\varepsilon}{\varepsilon_{1}}} d t_{2}\right. \\
& \left.+2 \int_{\varepsilon}^{1-\varepsilon} d t_{1} \int_{1-\varepsilon}^{\frac{1-\varepsilon}{1-\varepsilon t_{1}}} d t_{2}\right) t_{1}^{-1}\left(1-t_{1}\right)^{-1} t_{2}{ }^{-1}\left(1-t_{2}\right)^{-1} \\
& =\left((\ln \varepsilon)^{2}\right)+\left(\psi^{\prime}(1)\right)+\left((\ln \varepsilon)^{2}+2 \psi^{\prime}(1)\right) \\
& =5(\ln \varepsilon)^{2}+3 \psi^{\prime}(1)
\end{aligned}
$$

Hence the divergence cancels as expected, and we are left with the final result 
for the contact term, which is

$$
E_{5}\left(x_{1} x_{2} x_{3} x_{4} x_{5}\right) \underset{x_{i} \rightarrow 0}{\rightarrow} 3 \psi^{\prime}(1) \text {. }
$$

\section{The Lagrangian Density to order $\left(\alpha^{\prime}\right)^{2}$}

We now wish to obtain the Lagrangian density which reproduces correctly. the terms up to order $\left(\alpha^{\prime}\right)^{2}$ in $B_{5}$. For this purpose, we find that it is necessary to add to $L_{2}\left(\right.$ Eqn. 2.24) a contact term $\left(\phi^{5}\right)$ so that the complete contribution to $L$ up to order $\left(\alpha^{\prime}\right)^{2}$ is given by

$$
L_{2}^{2}=-\psi(I) \lambda^{2} \alpha^{\prime 2}\left[\frac{1}{4}(\partial \mu \phi)^{2}+\frac{1}{4}\left(\partial^{2} \mu \phi\right) \phi^{3}+\frac{1}{8} m^{2} \phi^{4}\right]+\frac{9}{5 l} \lambda^{3} \alpha^{\prime 2} \psi^{(1)} \phi^{5} \text {. }
$$

It is straightforward to show that the first term in (3.28) when combined with the $\frac{\lambda}{6} \phi^{3}$ part of the lagrangian gives the following contribution to the five point function:

$$
\psi^{(1)} \lambda^{3} \alpha^{2}\left[\sum_{\substack{i, j \\ i \neq j}} \frac{m^{2}}{m^{2}-\left(p_{i}+p_{j}\right)^{2}}-10\right]
$$

This contribution agrees precisely with the single pole contributions arising from the terms $\sum \frac{1}{x_{i}} B\left(x_{j}, x_{k}\right)$ (Eqn. 3.3) sumned over all inequivalent permutations.

$$
\begin{aligned}
& \text { More explicitly } \\
& \frac{\vdots}{x_{1}} B\left(x_{3}, x_{4}\right)=\frac{1}{x_{1}} \cdot\left(\frac{1}{x_{3}}+\frac{1}{x_{4}}\right) \cdots \psi^{\prime}(1) \frac{\left(x_{3}+x_{4}\right)}{x_{1}}+\ldots . .
\end{aligned}
$$

The first set of terms are correctly reproduced by $\frac{\lambda}{6} \phi^{3}$ calculated in the second order. The second set of terms coming from all inequivalent permutations lead to $(3.29)$.

The $\phi^{5}$ term in (3.28) is necessary to reproduce the contact terms $(3.27)$

This now explains why we wrote the $\phi^{4}$ derivative term in precisely the form (2.24) in the previous section, rather than any of the alternative forms noted in expression (2.25). The reason is that one requires that this part 
of $L_{2}$ reproduce correctly the contributions, $\left[\frac{1}{x_{i}} B\left(x_{j}, x_{k}\right)\right.$ of the single poles In $B_{5}$. This latter condition is, of course, the bootstrap condition that the Internal ground state poles consistently factorise into Born amplitudes for external ground state particles.

Note that if we did not impose the bootstrap requirement in this way, we could choose the (simple) form $\frac{1}{24} \psi^{(1)} \lambda^{2} \alpha^{\prime} \mathrm{m}^{2} \phi^{4}$ from (2.25) whereupon the corresponding $L^{\prime} 2$ is given by ${ }^{9}$

$$
L_{2}=\frac{1}{4 !} \psi^{(1)} \lambda^{2} \alpha^{\prime 2} m^{2} \phi^{4}-\frac{1}{5 !} \psi^{(1)} \lambda^{3} \alpha^{12} \phi^{5} .
$$

Although this form (3.31) happens to be simpler, and hence more attractive, It is not expected to have a simple generalisation to higher multiparticle. functions.

We should add that calculations along the above lines can be easily extended to six and higher-point functions. The computations are, however, very lengthy and no simple answer has emerged as yet.

\section{CONCLUDING REMARKS}

We have investigated the expansion of the four point function $B\left(-\alpha_{s},-\alpha_{t}\right)$ In the Regge slope parameter $\alpha^{\prime}$ with the hope of finding a Lagrangian density wh1ch goes beyond the zero-slope limit of Scherk ${ }^{1}$. Such a Lagrangian density can be written down formally by a Larent-Taylor series expansion of $B\left(-\alpha_{s},-\alpha_{t}\right)$ around the ground state pole $\alpha_{s}=a_{t}=0$. Such a formal Lagrangian written In terms of the scalar field $\phi$ is an infinite Lagrangian containing an infinite number of derivatives. This essential feature persists even in the drastic approximation of retaining only the next excited state beyond the ground state. Thus the Lagrangian is inherently non local and only in the strict zero slope Ilmit it reduces to the simple local $\phi^{3}$ Lagrangian. The nonlocality is not too surprising when one thinks of the arbitrary high mass and high spin states Included in the dual models. It is also expected if one believes, for example, 
In the ldea that the underlying internal hadronic structure is that of an extended relat1vistic massless string, embedded in space-time 10 .

We have also studied the higher order terms in the five-point function (order $\alpha^{\prime 2}$ ). We have shown that, under certain requirements, the Lagrangian necessary to describe all the terms to this order needs a $\phi^{5}$ contact term. Study of higher point functions with the possibility of finding some simple generalization is currently under progress.

In concluding, we would like to add one rather speculative remark concerning the renormalizability of the Lagrangian truncated at order $\alpha^{\prime 2}$; the Lagrangian $L=L_{0}+L_{1}+L_{2}$ leads to a non-renormalizable theory according to the conventional rules because of the presence of the $\phi^{5}$ contact term. We recall, however, that the dual model itself can lead to finite results if momentum-dependent counter terms are subtracted ${ }^{1} \cdot$. This raises the very Interesting possibility of a renormalization scheme for the Lagrangian $L$ by using techniques similar to those used in the renormalization of dual models. This needs further investigation. 


\section{Rarnzences end Footnotes}

i. J. Scherk, Nuelear Phrades 23!, 222 (1972): see also R. Sawyer, Proceedings of the Boulder Conference en Alch Enere Physics, 1969, page 419; N. Nakanishi, Prog. Theor. Prose. 48, 355 (2972).

2. A. Neveu and J. Seherk, Huezoar Paysics B36, 155 (1972).

3. P. H. Franpton; Phrsieal Roview DI, Nay 251973 and Syracuse University preprints SU-L205-12 and Ev-4205-14; A. P. Balechandran and H. Rupertsberger, Syracuse Univorst ty propelnt 8u-4205-28.

4. M. Abramowitz and J. A. Bterm. Handoook of Nathemetical Functions, Dover (1965) page 823, (Fai a Bruno!s formula):

5. The subscript in $f_{k}$ refpre to the arder in a' relative to the zero-slope

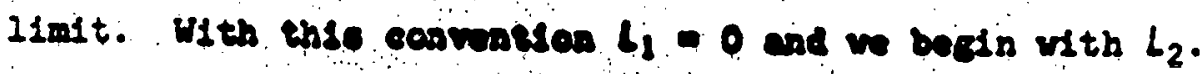

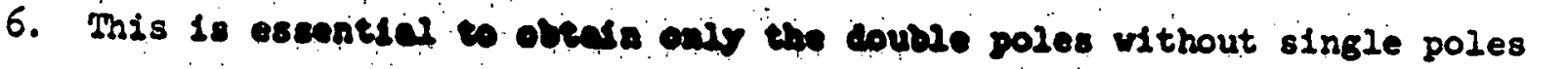
at $x, y=-2$ :

7. We have soan thot the concect tern is apont for $N=4$. This is actually an "accidental" propory or sh sure o suction, that can be related for

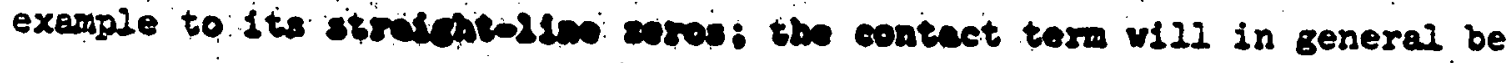
present, as dssevead blow. sor $N$. and oven at $N=4$ in more realistic Born emplitudes.

8. For rather an extanglve blallocrophy on cual models in general and their multiparticle extenalons in paticular see, for example, the ends of the various sections of P. D. Trapton, Lecture dotes on Dual Resonance Models

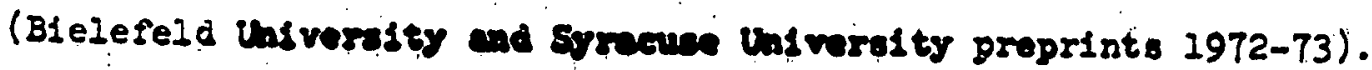

9. It is perhaps worth remurkson that on cen even sind a linear combination of the, alternate form in (2.25) that obvlates the need for any $\phi^{5}$ Interaction at the 85 level. of the expense of a compliceted (ad hoc) $\phi^{4}$ term; such a cholce, howevat. is of cource not consistent with our ldea of maintaining bootstrep conslstency order by order. 

10. Y. Nambu, Lecture notes prepared for the Summer Institute of the Niels Bohr Institute (SINBI) Copenhagen 1970; L. N. Chang and F. Mansouri, Physical Review D5 2535 (1972); P, Godderd,.J. Goldstone, C. Rebbi and C. B. Thorn Nuclear Physics $\underline{B}$ (to be published):

II. A. Neveu and J. Scherk, Physical Review DI, 2355 (1970), D. J. Gross, A. Neveu, J. Scherk and J. H. Schwartz, Physical Review D2, 697 (1970). 


\begin{tabular}{|c|c|c|c|c|}
\hline $\begin{array}{c}\text { Born Amplitude } \\
\text { to which } \\
\text { Term Contributes }\end{array}$ & $\begin{array}{c}\text { Zero-Slope } \\
\text { Limit }\end{array}$ & $O\left(\alpha^{\prime}\right)$ & $O\left(\alpha^{\prime 2}\right)$ & $O\left(\alpha^{\prime 3}\right)$ \\
\cline { 3 - 5 } & $\lambda \phi^{3}$ & {$\left[\lambda^{2} \alpha^{\prime} \phi^{4}\right]$} & $\lambda^{2} \alpha^{\prime 2}$ term & $\lambda^{2} \alpha^{3}$ term \\
$B_{5}$ & & $\lambda^{3} \alpha^{\prime 2} \phi^{5}$ & $\lambda^{3} \alpha^{3}$ term \\
$B_{6}$ & & $\lambda^{4} \alpha^{3} \phi^{6}$ \\
\hline
\end{tabular}

TABLE 1.

Terms in the Lagrangian density.

We refer to the $\lambda \phi^{3}$ term as the zero-slope limit, the terms $\lambda^{N-2}\left(\alpha^{\prime}\right)^{N-3} \phi^{N}(N \geq 4)$ as genuine contact terms, and aif others, which must involve derivatives or powers of $\mu^{2}$ or both, collectively as derivative terms. 


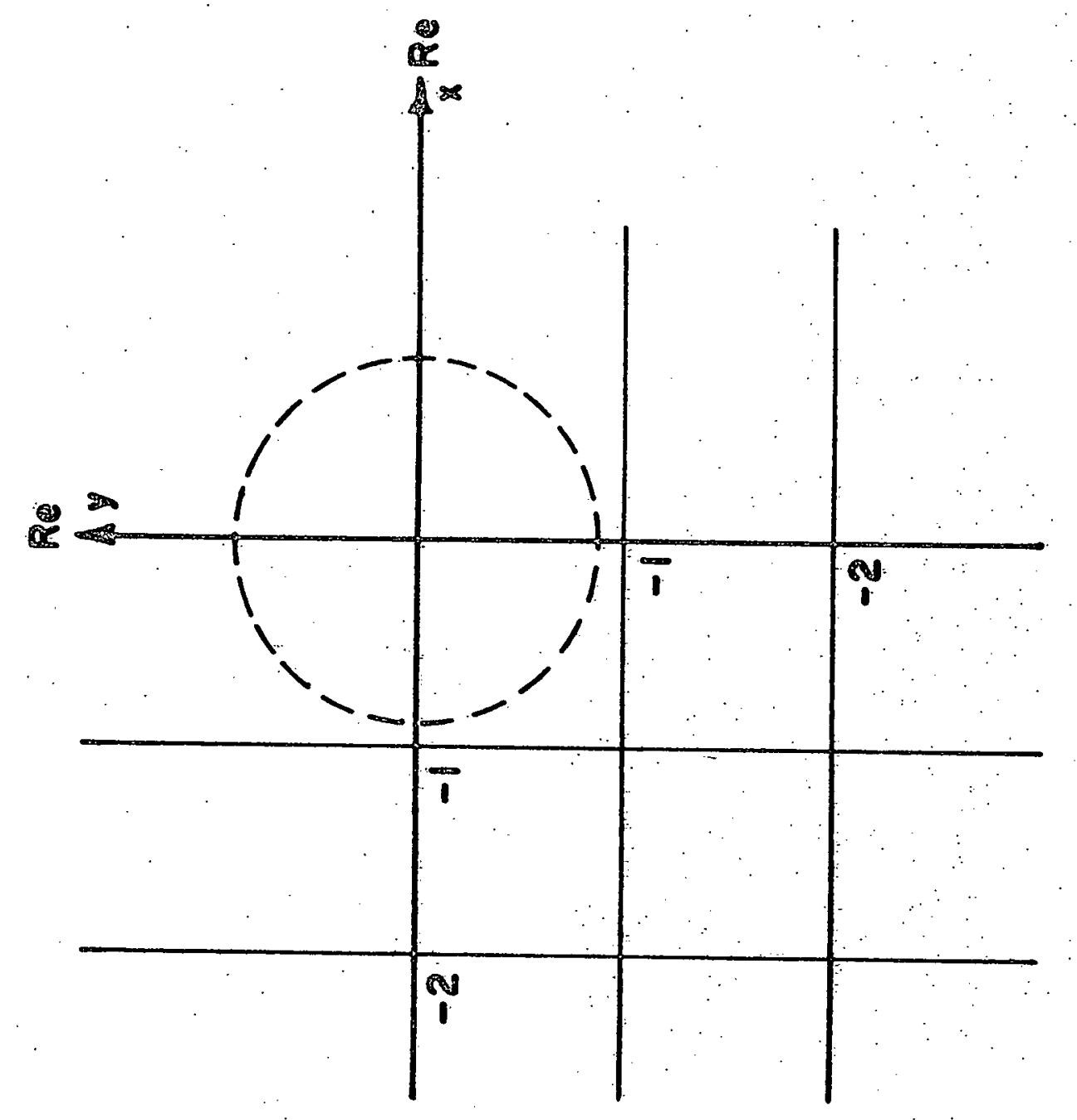

$\frac{1}{x}$
$\frac{5}{4}$ 

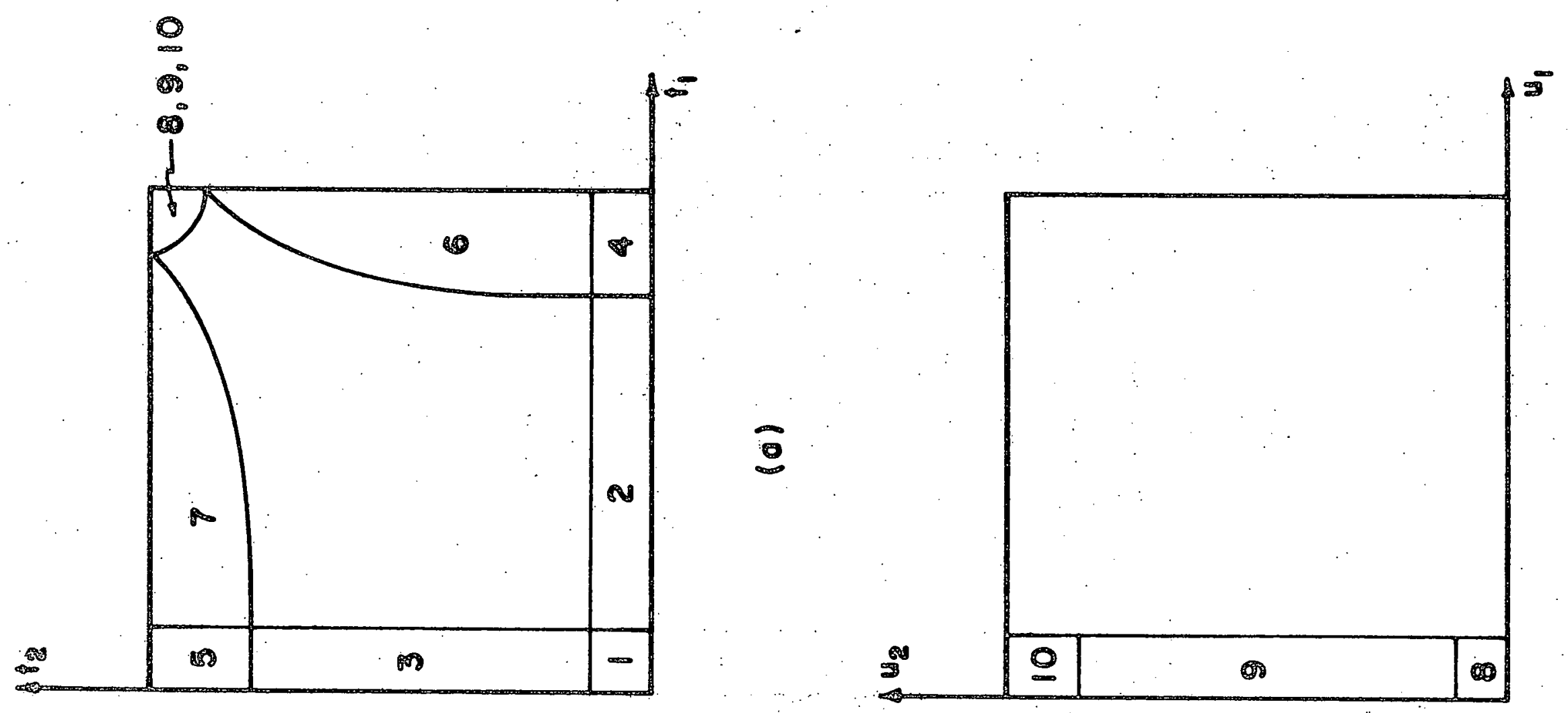

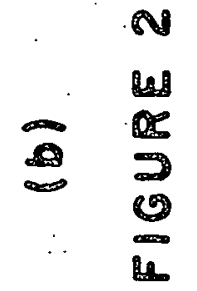





\section{Figure Captions}

Fig. 1. Region of analyticity in Re $x-$ Re $y$ plane.

Fig. 2. Subdivisions of integration region for $B_{5}$. 
I 